\title{
Substrate stiffness regulates extracellular matrix deposition by alveolar epithelial cells
}

\author{
This article was published in the following Dove Press journal: \\ Research and Reports in Biology \\ I 8 January 201 I \\ Number of times this article has been viewed
}

\author{
Jessica $L$ Eisenberg ${ }^{1,2}$ \\ Asmahan Safi ${ }^{3}$ \\ Xiaoding $\mathrm{Wei}^{3}$ \\ Horacio D Espinosa ${ }^{3}$ \\ GR Scott Budinger ${ }^{2}$ \\ Desire Takawira' \\ Susan B Hopkinson' \\ Jonathan $C R$ Jones ${ }^{1,2}$ \\ 'Department of Cell and Molecular \\ Biology, ${ }^{2}$ Division of Pulmonary \\ Medicine, Feinberg School of \\ Medicine, Northwestern University, \\ Chicago, IL, USA; ${ }^{3}$ Department of \\ Mechanical Engineering, Northwestern \\ University, Evanston, IL, USA
}

Correspondence: Jonathan $\mathrm{CR}$ Jones Department of Cell and Molecular Biology, Feinberg School of Medicine, Northwestern University,

303 East Chicago Avenue,

Chicago, IL 606II, USA

$\mathrm{Tel}+|3| 2503|4| 2$

Fax +I 3125036475

Email j-jones3@northwestern.edu
Aim: The aim of the study was to address whether a stiff substrate, a model for pulmonary fibrosis, is responsible for inducing changes in the phenotype of alveolar epithelial cells (AEC) in the lung, including their deposition and organization of extracellular matrix (ECM) proteins.

Methods: Freshly isolated lung AEC from male Sprague Dawley rats were seeded onto polyacrylamide gel substrates of varying stiffness and analyzed for expression and organization of adhesion, cytoskeletal, differentiation, and ECM components by Western immunoblotting and confocal immunofluorescence microscopy.

Results: We observed that substrate stiffness influences cell morphology and the organization of focal adhesions and the actin cytoskeleton. Surprisingly, however, we found that substrate stiffness has no influence on the differentiation of type II into type I AEC, nor does increased substrate stiffness lead to an epithelial-mesenchymal transition. In contrast, our data indicate that substrate stiffness regulates the expression of the $\alpha 3$ laminin subunit by AEC and the organization of both fibronectin and laminin in their ECM.

Conclusions: An increase in substrate stiffness leads to enhanced laminin and fibronectin assembly into fibrils, which likely contributes to the disease phenotype in the fibrotic lung.

Keywords: alveolar epithelial cells, fibrosis, extracellular matrix, substrate stiffness

\section{Introduction}

The lung alveoli are air sacs that permit oxygen exchange between the air space and the blood. The alveolar wall is covered by epithelial cells (alveolar epithelial cells, AEC) comprised mainly of flattened type I cells that cover $\sim 95 \%$ of the alveolar surface area and cuboidal type II cells. ${ }^{1-4}$ Type I cells interfaced with pulmonary capillaries are responsible for gas exchange and are susceptible to oxidant stress. Type II cells produce surfactant, proliferate, differentiate into type I cells, and have stem cell-like properties.

The extracellular matrix (ECM) plays a substantial role in maintaining proper adhesion, proliferation, and differentiation of various cell types, with AEC being no exception. In vitro studies have revealed that AEC secrete a complex, fibrillar matrix composed of several components (specifically, perlecan, nidogen, and laminin), but their matrix is uniquely rich in laminin-311 (formerly known as laminin 6). ${ }^{5-7}$ Laminin-311 in this matrix assembles in an integrin-dependent manner, and there is also evidence that it is involved in mechanosignaling induced by stretch., ${ }^{8,9}$

The ECM is known to be an important regulator of alveolar epithelial development and functions in both normal and diseased lungs. ${ }^{10,11}$ For example, in pulmonary fibrosis, a disease afflicting over 200,000 people in the US, aberrant accumulation 
of a collagen-rich ECM in the lung parenchyma results in tissue stiffening and a progressive decline in lung function due to modulation of AEC phenotype. ${ }^{12-15}$ Additionally, some in vivo and in vitro models suggest that the decline in lung function in fibrotic disease involves an extracellular matrix/ growth factor-regulated AEC epithelial-mesenchymal transition (EMT). ${ }^{16-18}$

Substrate stiffness has a profound influence on the adhesion, migration, proliferation, and differentiation of numerous cell types, including cardiomyocytes, adult neurons, stem cells, vascular smooth muscle cells, human umbilical artery endothelial cells, fibroblasts, osteoblasts, and cervical epithelial cells. ${ }^{19-26}$ However, the role of substrate stiffness versus ECM deposition in regulating AEC phenotype in the normal and the fibrotic lungs has not been addressed experimentally. In this study, we assessed how adhesion, differentiation, EMT, and matrix deposition of AEC differ on substrates with defined stiffness values. Specifically, we evaluated the consequences of maintaining AEC on polyacrylamide gel substrates approximating a range of stiffness mimicking the values for normal and fibrotic tissues. ${ }^{19,27-31}$ Our results indicate that substrate stiffness does not directly regulate AEC differentiation or EMT, but does influence cell morphology as well as the deposition and organization of laminin within the ECM.

\section{Methods}

\section{Preparation of polyacrylamide gels}

Wang and Pelham's method ${ }^{32}$ was utilized to prepare polyacrylamide gel substrates with stiffness values mimicking those of the normal and fibrotic tissues..$^{19,27-31}$ Polyacrylamide gels ( $\sim 50 \mu \mathrm{m}$ thick, as determined by confocal microscopic scanning between the upper glass surface and the upper gel surface) of three different stiffness values (designated low, medium, and high) were prepared on aminosilanized glass coverslips by mixing three ratios of acrylamide and bis-acrylamide solutions (7.5\%:0.2\%, 7.5\%:0.35\%, and 12\%:0.6\%) with sterile water, $10 \%$ ammonium persulfate, and $N, N, N^{\prime}, N^{\prime}$ tetramethylethylenediamine (TEMED) (each from SigmaAldrich, St Louis, MO). Following polymerization, the gels were washed with several changes of $50 \mathrm{mM}$ HEPES buffer ( $\mathrm{pH} 8.5$ ) and then activated with $0.5 \mathrm{mg} / \mathrm{mL}$ sulfo-SANPAH (Pierce/Thermo Fisher Scientific, Rockford, IL) exposed to 25-30 min of ultraviolet light using a UV Stratalinker 1800 (Strategene, Santa Clara, CA). The gels were then washed twice with $50 \mathrm{mM}$ HEPES for $15 \mathrm{~min}$ each, followed by incubation at $4{ }^{\circ} \mathrm{C}$ with shaking for $18-24 \mathrm{~h}$ for covalent attachment of rat tail type I collagen (BD Biosciences, San Jose, CA).

\section{Nanoindentation analysis of polyacrylamide gels}

The stiffness of polyacrylamide gels was characterized by atomic force microscopy (AFM) based nanoindentation using a XE-120 AFM (Park Systems, Santa Clara, CA). ${ }^{33}$ The substrates, placed in a liquid cell with an integrated heating stage, were immersed in deionized water and maintained at $37^{\circ} \mathrm{C}$ throughout all experiments. Indentations were done using a spherical silicon oxide AFM probe integrated on a silicon nitride cantilever (CP-PNP-SiO sQube, Wetzlar, Germany). The diameter of each spherical probe was measured using scanning electron microscopy and ranged between 1.4 and $1.7 \mu \mathrm{m}$. A static deflection method, ${ }^{34}$ which employs a calibrated reference cantilever (CLFC-NOBO; Vecco Probe, Camerillo, CA), was used to determine the normal spring constants of the two cantilevers $(0.12$ and $0.2 \mathrm{~N} / \mathrm{m}$ ) used in this study. The deflection sensitivity was calibrated in water by recording a force-displacement curve on a silicon substrate prior to each indentation series. Displacement controlled load-displacement curves were obtained in the nanoindentation mode with a loading rate of $0.30 \mu \mathrm{m} / \mathrm{sec}$. A holding time of $3 \mathrm{sec}$ was applied in nanoindentation mode to reduce the viscoelastic effect before unloading. For this study, the technique described by Oliver and Pharr ${ }^{35}$ and a Hertzian model ${ }^{36}$ were employed to quantify the gel stiffness.

\section{$\mathrm{AEC}$ isolation and cell culture}

AEC were isolated from pathogen-free, male Sprague Dawley rats (200-225 g) as previously described. ${ }^{5,8,9}$ Briefly, the lungs were perfused via the pulmonary artery, lavaged, and digested with $3 \mathrm{U} / \mathrm{mL}$ elastase (Worthington Biochemical, Freehold, $\mathrm{NJ}$ ). AEC were then purified by differential adherence to dishes pretreated with immunoglobulin $\mathrm{G}$, and cell viability was assessed by trypan-blue exclusion (>95\%). Cells were resuspended in Dulbecco's modified Eagle's medium (Cellgro, Mediatech Inc, Herndon, CA) containing 10\% fetal bovine serum (FBS; Hyclone, Logan, UT) with $2 \mathrm{mM}$ L-glutamine, $100 \mathrm{U} / \mathrm{mL}$ penicillin, $100 \mathrm{mg} / \mathrm{mL}$ streptomycin, and $0.25 \mathrm{mg} / \mathrm{mL}$ amphotericin $\mathrm{B}$. The cells were seeded onto ECM-coated glass or polyacrylamide gel substrates within 12 -well culture plates at a density of 400,000 cells $/ \mathrm{mL}$. The day of the cell isolation is designated as day 0 in culture, and subsequent experiments were performed on days 2 and 5 . RLE-6TN cells, immortalized rat AEC, were obtained from the American Type Culture Collection (Manassas, VA, USA) and used as controls for immunofluorescence staining. The cells were cultured in Nutrient Mixture F-12 Ham 
medium (Sigma-Aldrich) containing 10\% FBS with $2 \mathrm{mM}$ L-glutamine, $100 \mathrm{U} / \mathrm{mL}$ penicillin, $100 \mathrm{mg} / \mathrm{mL}$ streptomycin, and $0.25 \mathrm{mg} / \mathrm{mL}$ amphotericin $\mathrm{B}$. All cells were maintained in a humidified chamber at $37^{\circ} \mathrm{C}$ with $5 \% \mathrm{CO}_{2}$.

\section{Antibodies and other reagents}

A polyclonal antibody against prosurfactant protein $\mathrm{C}$ (SP-C) was purchased from Chemicon/Millipore (Billerica, MA), a monoclonal antibody against $\mathrm{T} 1 \alpha$ was generously provided by Dr Leland Dobbs from the University of California, San Francisco, a monoclonal antibody against vimentin was purchased from BD Biosciences (clone RV202), and a rabbit polyclonal antibody against alpha-smooth muscle actin ( $\alpha$-SMA) was purchased from Epitomics (Burlingame, CA, USA). A rabbit antibody against pan keratin was the kind gift of Dr Robert D Goldman (Northwestern University, Evanston, IL). The mouse monoclonal antibody 10B5 against the $\alpha 3$-subunit of laminin has been previously described. ${ }^{37}$ A polyclonal antibody against lamin $\mathrm{A} / \mathrm{C}$ was purchased from Cell Signaling Technology (Danvers, MA). A rabbit polyclonal fibronectin (FN) antibody and mouse monoclonal against vinculin were obtained from Sigma-Aldrich. Rhodamineconjugated phalloidin and 4',6-diamidino-2-phenylindole (DAPI) were purchased from Molecular Probes (Eugene, OR). Horse radish peroxide-conjugated secondary antibodies for Western blotting and fluorescently labeled secondary antibodies for immunofluorescence were purchased from Jackson ImmunoResearch Laboratories (West Grove, PA).

\section{SDS-PAGE and immunoblotting}

AEC were solubilized in sample buffer consisting of $8 \mathrm{M}$ urea, $1 \%$ sodium dodecyl sulfate in $10 \mathrm{mM}$ Tris- $\mathrm{HCl}(\mathrm{pH}$ 6.8), and 10\% $\beta$-mercaptoethanol (Sigma-Aldrich). Proteins were separated by SDS-PAGE, transferred to nitrocellulose membranes, and processed for immunoblotting as previously described. ${ }^{9}$ Immunoblots were scanned and quantified using ImageJ (National Institutes of Health, Bethesda, MD). Results from a minimum of three blots, using lysates from at least two independent cell isolations, were combined and presented as the mean \pm standard deviation. Significant differences between experimental conditions were explored with the two-tailed, paired Student's $t$-test and GraphPad Prism software (version 3.0; GraphPad Software, La Jolla, CA). The significant difference was $P<0.05$.

\section{Immunofluorescence and microscopy}

Live images of AEC on the substrates on days 2 and 5 following isolation were captured using a $20 \times$ objective on a Nikon TE2000 inverted microscope (Nikon Inc, Melville, NY). Substrates were then removed from culture plates and prepared for immunostaining. Two fixation protocols were used to achieve optimal staining. A fixation method using 3.7\% formaldehyde in phosphate-buffered saline (PBS) for $5 \mathrm{~min}$ at room temperature followed by $5 \mathrm{~min}$ of permeabilization with $0.3 \%$ Triton $\mathrm{X}-100$ in PBS was utilized for staining focal adhesion proteins and actin filaments. For matrix and vimentin/keratin staining, cells were fixed and extracted with $-20^{\circ} \mathrm{C}$ acetone for $2 \mathrm{~min}$ and air-dried as previously described. ${ }^{38}$ Fixed specimens were processed with primary antibodies at $37^{\circ} \mathrm{C}$ for $2 \mathrm{~h}$ and washed with multiple changes of $1 \%$ bovine serum albumin (BSA; SigmaAldrich) in PBS. They were then incubated for $1.5 \mathrm{~h}$ at room temperature with fluorescein- and rhodamine-conjugated secondary antibodies. All substrates were extensively washed with $1 \% \mathrm{BSA} / \mathrm{PBS}$ and then mounted onto slides for imaging. Immunofluorescence microscopy was performed in the Northwestern University Cell Imaging Facility using a Zeiss LSM 510 META laser scanning confocal microscope (Carl Zeiss, Thornwood, NY) with either a $100 \times$ objective or a $63 \times$ objective with $2 \times$ digital zoom. Images were exported as TIFF files and analyzed with ImageJ software (National Institutes of Health).

\section{Results \\ Stiffness characterization of the polyacrylamide gel substrates}

Based upon previously reported polyacrylamide preparations $\mathrm{s}^{20,32,39}$ as well as reported measurements of similar polyacrylamide gels using AFM, ${ }^{39-42}$ we generated low, medium, and high stiffness gels predicted to possess Young's modulus values of $\sim 5,10$, and $55 \mathrm{kPa}$, respectively. These values were chosen because they represent stiffnesses within the range of normal and fibrotic tissues reported in the literature. ${ }^{19,27-31}$ The Young's moduli of the polyacrylamide gels as a function of the molecular percentage of the bis-acrylamide cross-linker were characterized using AFM (Figure 1A). Average stiffness values of $12 \pm 2.95$ and $16 \pm 3 \mathrm{kPa}$ were found for the low (7.5\%:0.2\% acrylamide:bis-acrylamide) and the medium $(7.5 \%: 0.35 \%)$ cross-linked gels, respectively, somewhat higher than the predicted values, while the highly cross-linked (12\%:0.6\% acrylamide:bis-acrylamide) gels exhibited much higher stiffness $(51 \pm 12 \mathrm{kPa})$. For the low cross-linked gels, the stiffness was obtained by fitting a Hertzian linear contact model ${ }^{36}$ to the loading curve, while the medium and high cross-linked gel stiffness values were determined by the Oliver and Pharr $(\mathrm{O}-\mathrm{P})$ procedure. ${ }^{35}$ 
A

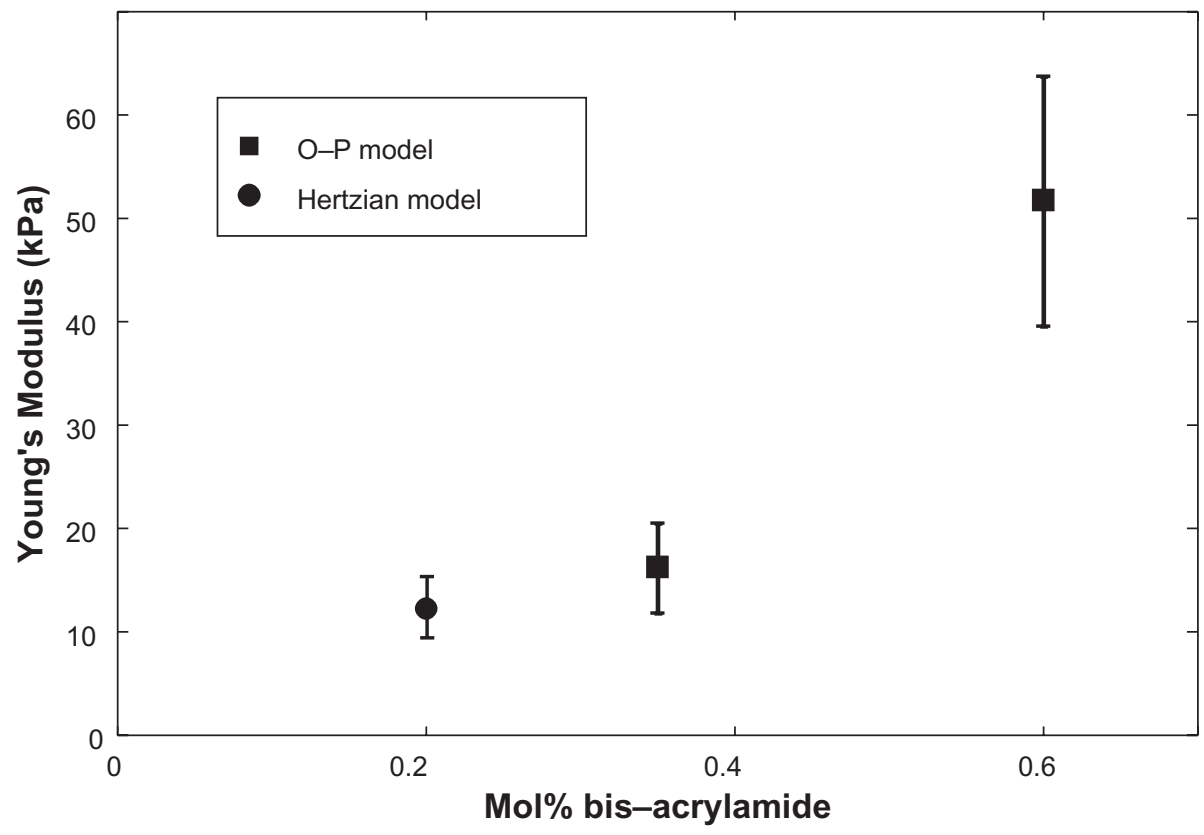

B

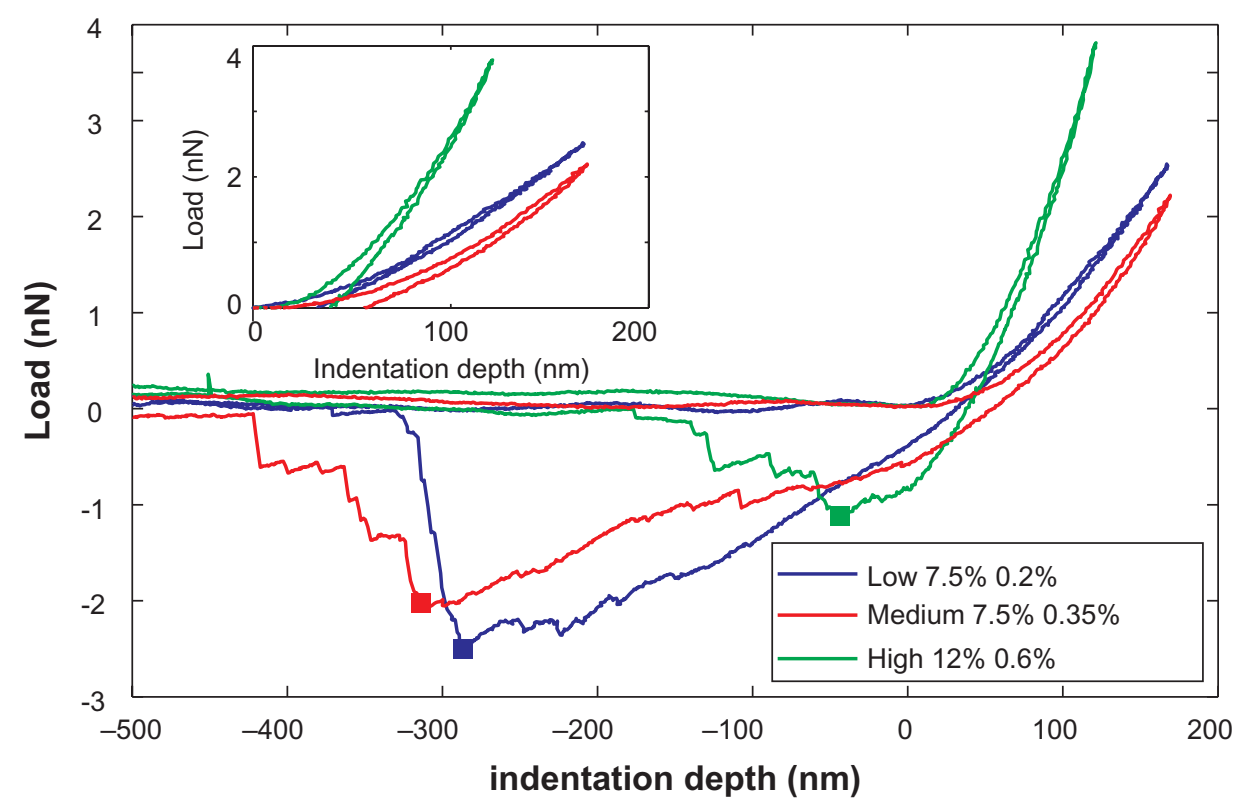

Figure I Characterization of polyacrylamide gel stiffness with mol\% acrylamide:bis-acrylamide composition of 7.5\%:0.2\% (low), 7.5\%:0.35\% (medium), and I2\%:0.6\% (high). A) Elastic moduli as function of bis-acrylamide cross-linker concentration for polyacrylamide measured by AFM indentation with O-P model (squares) and Hertzian linear model (circle). Error bars represent standard deviation for over 25 indents at various locations. B) Typical load-displacement curves for polyacrylamide gel with 0.2 , 0.35 , and $0.6 \mathrm{~mol} \%$ of cross-linker, respectively. Each curve consists of approaching, loading, unloading, and lifting up segments. Solid points on curves indicate the adhesion forces measured during retraction. Inset shows indentation segments used for stiffness characterization.

Abbreviations: AFM, atomic force microscopy; O-P, Oliver and Pharr.

The typical indentation curves for the three types of gels studied are also shown (Figure 1B). The pull-out forces (square dots in Figure 1B) during retraction increased with decreasing gel stiffness. This suggests that for the low crosslinked gel, pile-up is likely leading to an underestimation of contact area, ie, an overestimation of stiffness when the O-P method is used. Such an overestimation of stiffness for nanoindentation studies on polymeric material exhibiting viscoelastic behavior and pile-up has been discussed previously. ${ }^{43,44}$ Thus, a Hertzian model, which is less affected by pile-up as it considers only the beginning of the loading curve, was fitted to the low cross-linked gel indentation curves to identify its stiffness. The Hertzian fit for the low cross-linked gel gave a Young's modulus of $12 \mathrm{kPa}$, while the O-P method gave a value of $20 \mathrm{kPa}$. However, when applied to medium and high cross-linked gels, the Hertzian model 
does not accurately describe the measured load-displacement curves. Complex deformation mechanism such as viscoelasticity and nonlinear behavior during loading could explain why the Hertzian model does not apply in these cases.

The Young's moduli of high and medium cross-linked gels identified here are found to be in good agreement with previous work, which applied AFM indentation to study the mechanical behavior of polyacrylamide gels. ${ }^{20,41,43,45}$ However, Engler et al and Solon et al reported lower Young's modulus values for the same low bis-acrylamide concentration that we prepared. Such differences could arise from either the molecular structure of the polymer or the employed synthesis methods. Nonetheless, there was a consistent trend of increasing moduli by increasing the amount of cross-linker as reported previously. ${ }^{20,41,43}$

\section{The effect of stiffness on cell shape, focal adhesions, and the actin cytoskeleton of AEC}

Freshly isolated primary rat type II AEC were seeded onto polyacrylamide gel substrates of low, medium, and high stiffness, along with glass coverslips. The AEC remain relatively round on low and medium stiffness substrates after 2 days in culture, but were found to adopt a more flattened morphology on the high stiffness substrate and glass within the same time period (Figure 2A). By day 5 after isolation, the cells on each substrate appeared flattened, although cells on the stiffer substrates exhibited the most well-spread profiles (Figure 2A). We proceeded to quantify the areas occupied by 20 cells on the different substrates. These cells were randomly selected. We found a significant difference between the values of cells maintained on low and medium stiffness substrates compared to those of cells on the high stiffness substrate and glass at day 2 (Figure 2B). By day 5, AEC on each of the polyacrylamide gels were found to be significantly less well spread than those maintained on glass.

We also chose to look at the development of focal adhesions and the actin cytoskeleton since these structures are known to be influenced by substrate compliance. ${ }^{46-48}$ $\mathrm{AEC}$ at 2 and 5 days after isolation and plating were stained to visualize the focal adhesion protein vinculin and the actin cytoskeleton (Figure 2C). After 2 days in culture, we noted that cells on the lower stiffness substrates had smaller vinculin-containing focal adhesions, compared to the larger focal adhesions in the more flattened cells on the higher stiffness substrates (Figure 2C). We determined the average size of 20 random focal adhesions per cell in at least three different AEC on each substrate and found the observed differences in focal adhesion size to be statistically significant (Figure 2D). Fibers comprising the actin cytoskeletons in the cells after 2 days in culture were primarily located around the cell periphery for all substrates, although thicker bundles were present in cells maintained on the higher stiffness substrates (Figure 2C). By day 5, vinculin and actin cytoskeleton organization in cells on all substrates appeared similar, although the sizes of the focal adhesion in cells on each of the gels were determined to be significantly smaller than those on the glass substrate (Figure 2D).

\section{The effect of stiffness on AEC differentiation and EMT}

In vitro, AEC undergo a differentiation from type II to type I cells. ${ }^{1-4}$ As a consequence, there is a loss in expression of type II cells markers (such as SP-C) with a concomitant upregulation in expression of type I AEC markers (including T1 $\alpha){ }^{8}$ Therefore, to assess whether substrate stiffness has an effect on type I differentiation, we collected lysates from the freshly isolated AEC or AEC seeded onto the different substrates at 2 and 5 days after plating and probed these lysates with antibodies against SP-C and T1 $\alpha$. Surprisingly, despite the observed differences in cell spreading, we saw a loss in the expression of SP-C within 2 days of plating and a concomitant increase in $\mathrm{T} 1 \alpha$ expression, the level of which remains unchanged between days 2 and 5 in culture, regardless of substrate stiffness (Figure 3).

Several studies also indicate that pulmonary fibrosis, which results in the scarring and stiffening of lung tissue, is partially mediated by AEC EMT. ${ }^{16-18}$ Generally, EMT occurs when an extracellular cue, such as tissue inflammation, causes the upregulation of a particular growth factor or transcription factor that signals the epithelial cells to transform into a mesenchymal, or fibroblast, cell type. ${ }^{49,50}$ Therefore, we next determined if substrate stiffness would influence EMT in the AEC. Lysates derived from freshly isolated AEC or from AEC maintained for 2 and 5 days on substrates of varying stiffness were probed with antibodies against vimentin and $\alpha$-SMA (Figure 3A, B). Although we detected vimentin and $\alpha$-SMA in extracts of day 0 AEC suggesting a potential fibroblast contamination in our primary epithelial cell isolates, $>90 \%$ of the adherent AEC at day 5 were stained by an antibody against keratin, a marker of epithelial cell phenotype (Figure 3A, C). Moreover, regardless of substrate stiffness, the levels of expression of both vimentin and $\alpha$-SMA in the lysates of AEC cultured for 2 and 5 days after isolation did not differ substantially, indicating that the cells did not undergo EMT (Figure 3). 
A
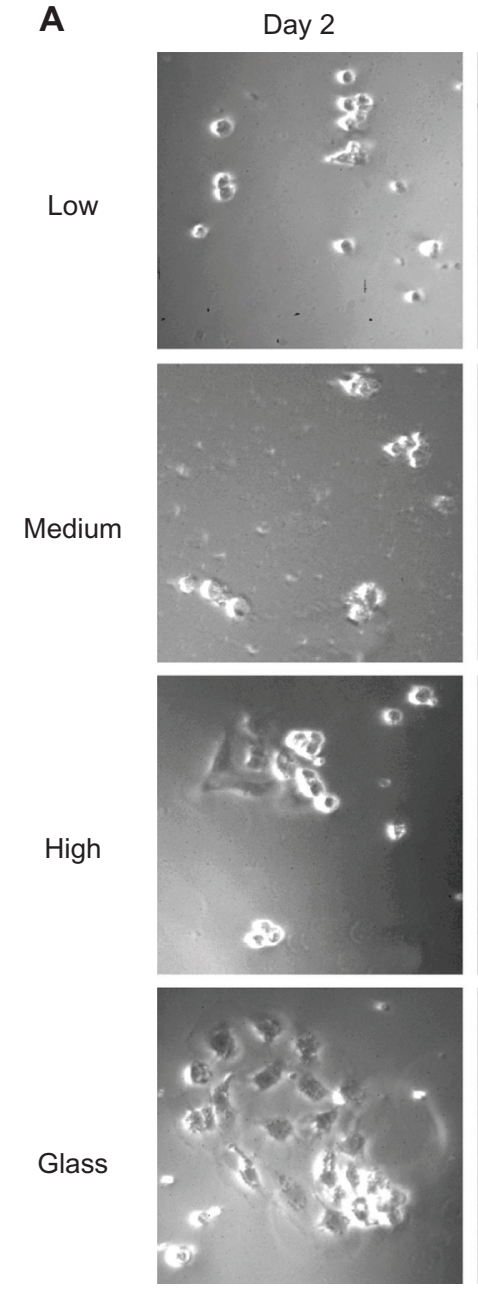

B

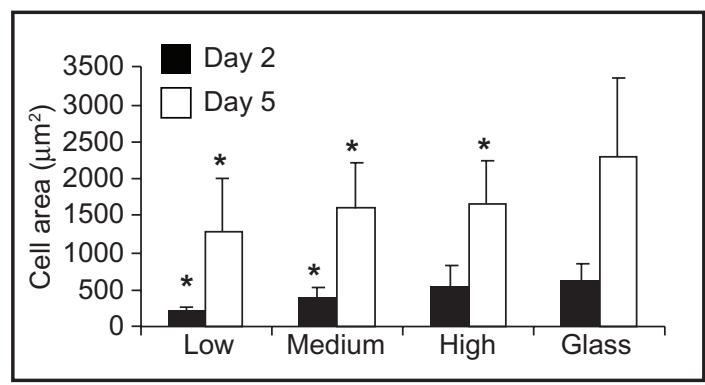

Day 5
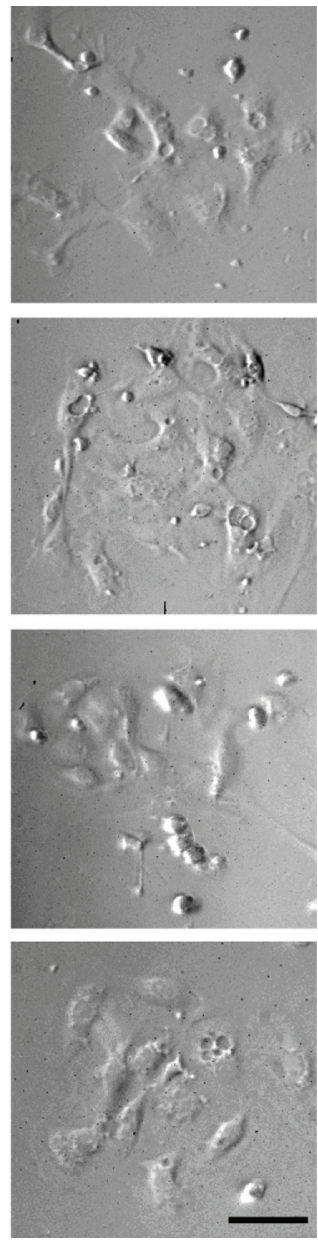

C
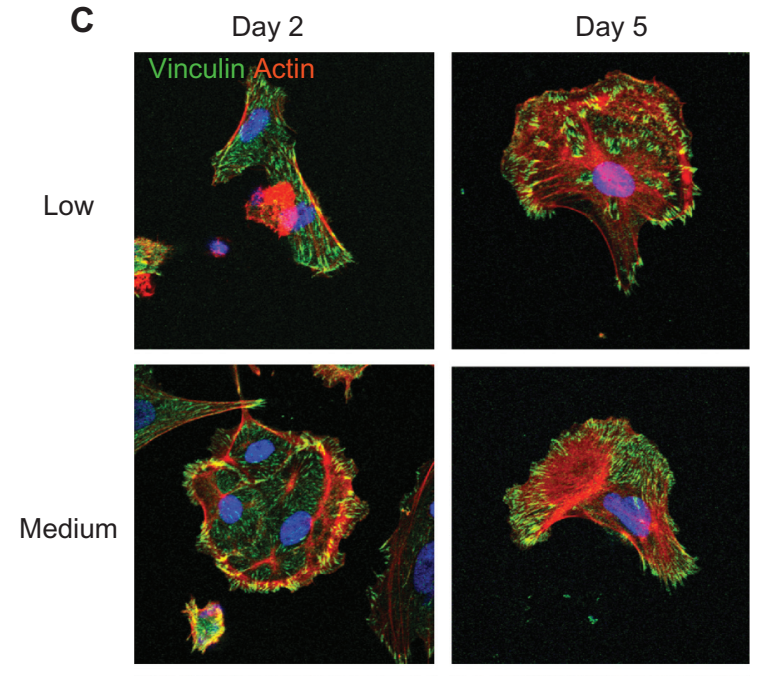

High
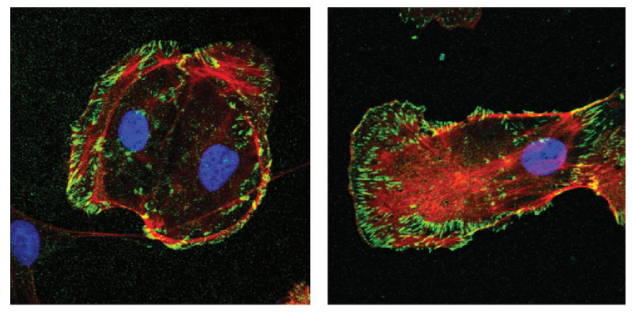

Glass
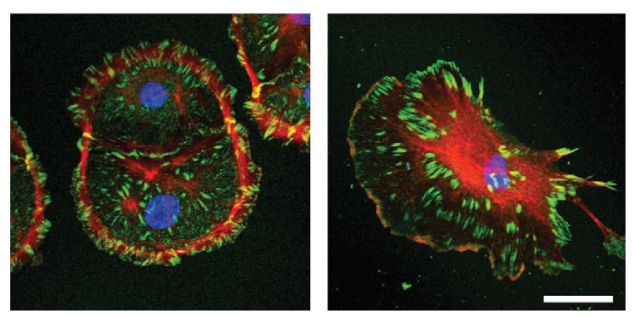

D

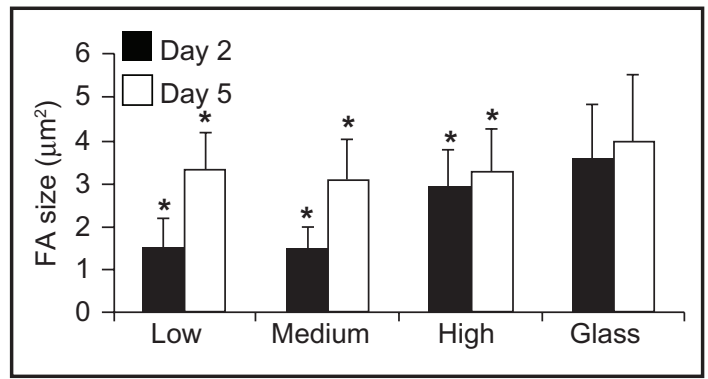

Figure 2 A) Representative images of live AEC cultured for 2 and 5 days on low, medium, and high gels, as well as glass substrates reveal changes in cell morphology. B) Quantification of the cell areas was performed on 20 cells attached to each substrate. C) AEC were also fixed and immunostained after 2 and 5 days in order to visualize the focal adhesion protein vinculin (green), the actin cytoskeleton (red), and the cell nucleus (blue). D) Quantification of focal adhesion size was done by measuring 20 random focal adhesions in 3 different cells on each substrate.

Notes: Scale bar for live cells in (A) represents $50 \mu \mathrm{m}$, while the bar in (C) represents $20 \mu \mathrm{m}$. *P $<0.05$ significance compared to the respective glass substrate.

Abbreviations: AEC, alveolar epithelial cells, FA, focal adhesion.

There was some variability in vimentin expression in the cells maintained on different substrates, possibly resulting from differences in epithelial purity in the adherent population. However, there was no consistent trend in this result. Interestingly, although immunoblotting reveals that our AEC express vimentin, regardless of substrate stiffness, no obvious vimentin filamentous staining is observed in our cell populations. In contrast, immortalized rat AEC exhibit an extensive filament network of vimentin but show little, if any, keratin staining (Figure 3A, C). These data suggest that vimentin in primary cultured AEC may be in a soluble, nonfilamentous form. 
A

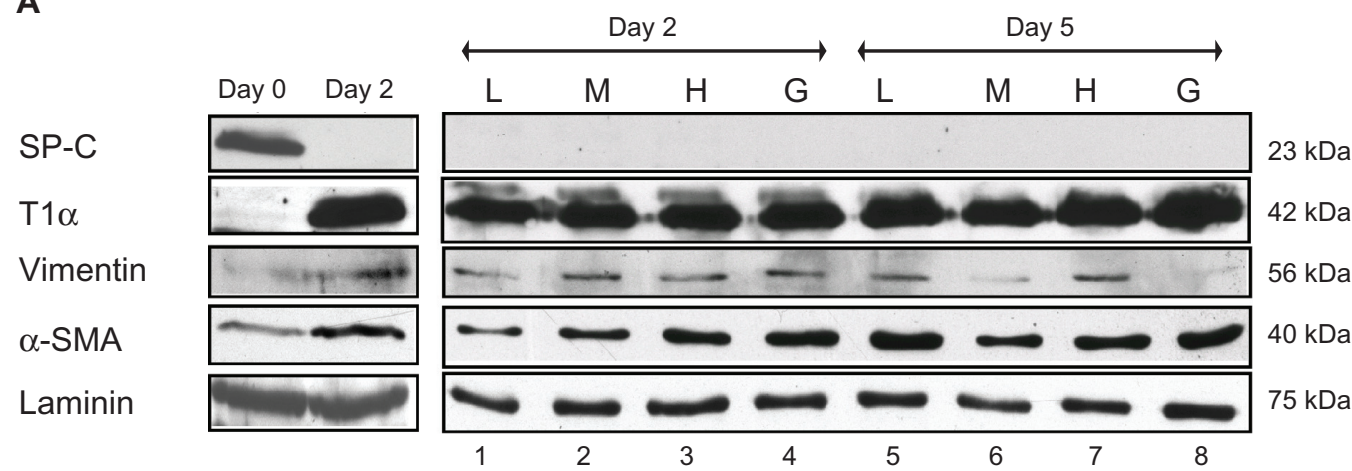

B

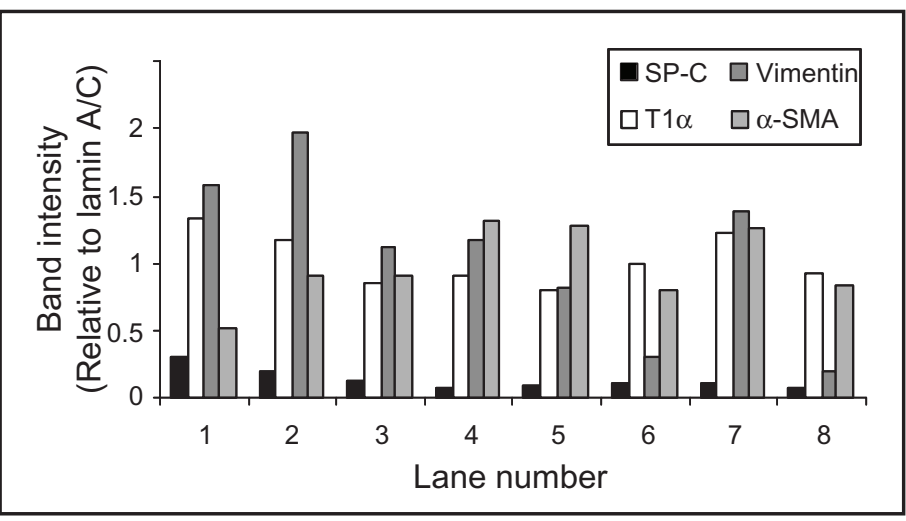

C

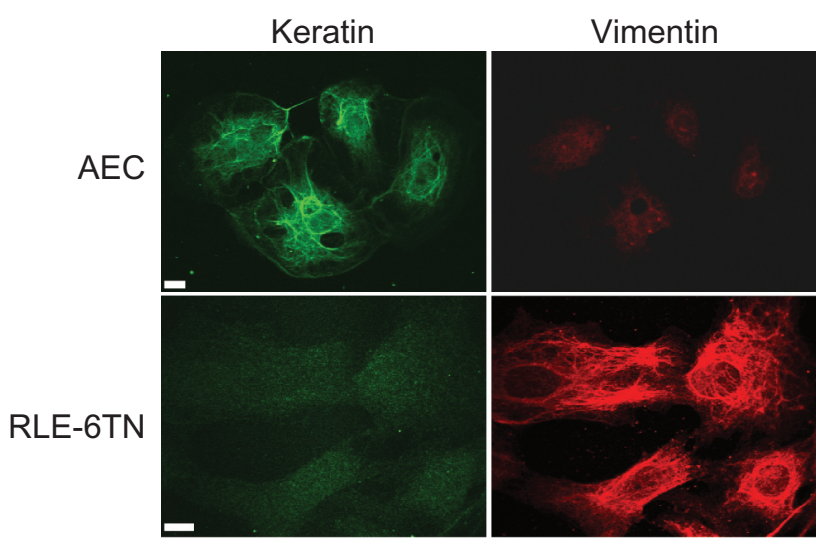

Figure 3 A) Western blots were run on AEC lysates to examine differentiation and EMT. The lysate from freshly isolated AEC (day 0) was processed for blotting together with an extract derived from AEC cultured for 2 days (day 2) on glass. Day 0 cells are positive for the AEC type II marker SP-C, but negative for the type I AEC marker TI $\alpha$. The reverse is the case for day 2 cells. B) Western blots for the differentiation and EMT markers in lysates from AEC cultured on low, medium, and high gels, as well as glass substrates for 2 and 5 days postisolation (lanes I-8), and the quantification of the blots indicates that substrate stiffness does not appear to determine differentiation or EMT. C) To determine the purity of the isolated AEC, the cells were fixed and stained for keratin and vimentin on day 5 . The AEC stain is positive for keratin, an epithelial cell marker, but is negative for vimentin, a fibroblast protein marker. The RLE-6TN cell line was used as a positive control for vimentin antibody reactivity. Scale bars I0 $\mu$ m. Abbreviations: AEC, alveolar epithelial cells; EMT epithelial-mesenchymal transition; RLE-6TN, immortalized rat lung epithelial cell line; L, low stiffness gel; M, medium stiffness gel; $\mathrm{H}$, high stiffness gel; $\mathrm{G}$, glass substrate; SP-C, prosurfactant protein-C; $\alpha$-SMA, alpha-smooth muscle actin.

\section{Stiffness influences matrix expression and deposition by AEC}

We reported earlier that AEC deposit a fibrillar network of laminin-311 in culture and that this deposition correlates with cell spreading. ${ }^{5,8}$ We also suggested that laminin-311 deposition and assembly of laminin-311 fibrils is a feature characteristic of type I cells. Interestingly, AEC also deposit a network of fibronectin (FN) fibrils when maintained on glass (Figure 4). Moreover, laminin-311 and FN fibrils not only exhibit colocalization in the matrix of AEC, but the deposition and assembly of FN fibrils also precedes that of laminin-311. We wondered if the deposition of FN and/or 


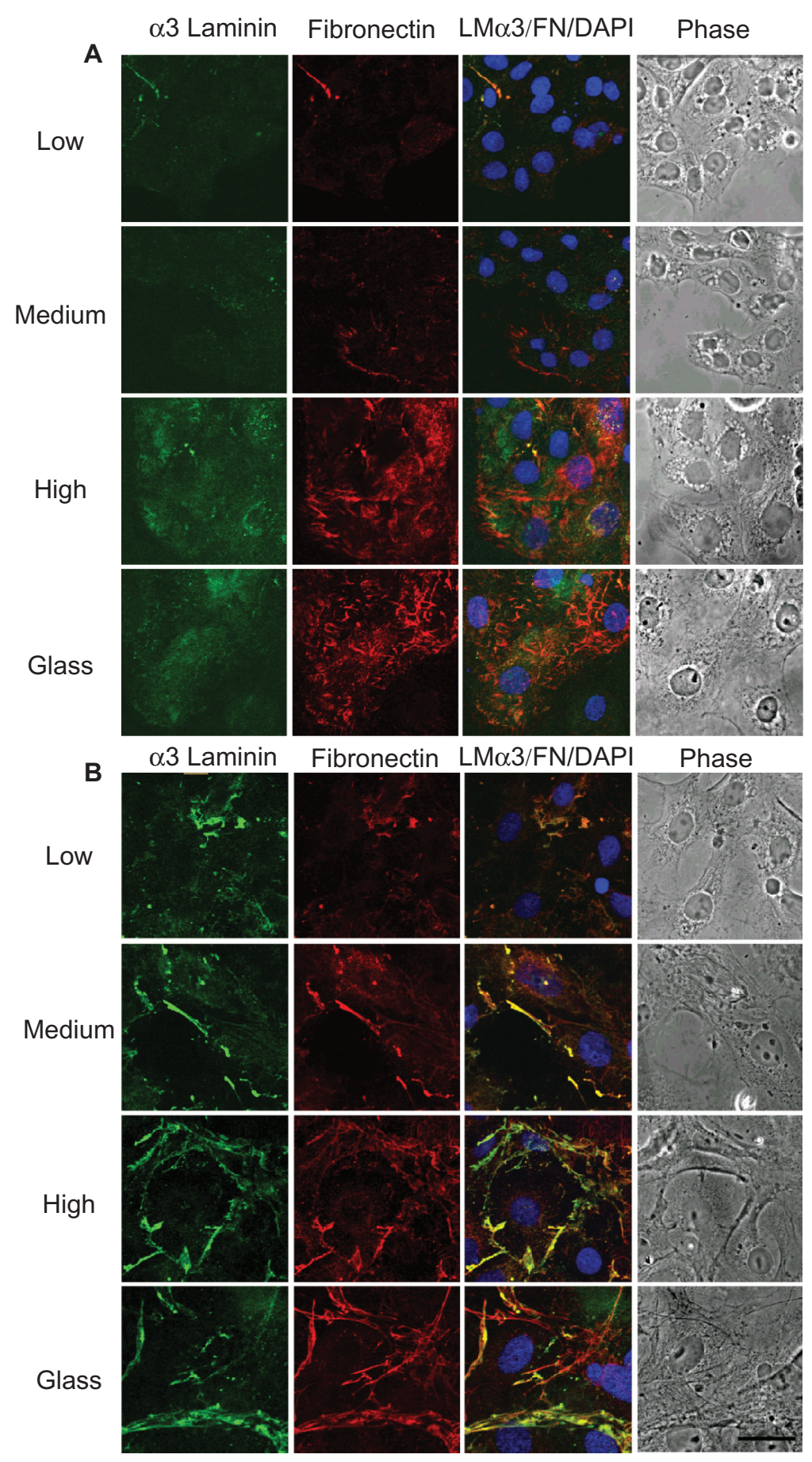

Figure 4 Representative images of cultured AEC that were fixed and stained to visualize the $\alpha 3$ laminin subunit, fibronectin, cell nucleus (DAPI), as well as phase contrast for cells that had attached to substrates for A) 2 days and B) 5 days postisolation

Note: Scale bar shown in (B) applies to all images and represents $20 \mu \mathrm{m}$.

Abbreviations: LM $\alpha 3$, alpha3 laminin subunit; FN, fibronectin; DAPI, 4',6-diamidino-2-phenylindole.

laminin-311 is regulated by substrate stiffness. After 2 days in culture, we found that the $\alpha 3$ laminin subunit antibody failed to stain the AEC matrix (Figure 4A). In contrast, at the same time point, either the matrix was diffusely stained by FN antibodies or short fibers of FN were observed in the matrix of AEC, with the more prevalent fibers seen with cells maintained on stiffer substrates (Figure 4A). However, after 5 days in culture, we observed extensive colocalization of the $\alpha 3$ laminin subunit with FN-rich fibrils as well as a significant increase in the length of fibrils in the matrix of cells 
maintained on stiffer substrates (Figure 4B). In addition, we failed to observe staining of the $\alpha 3$ laminin subunit where no FN fibrils were present, supporting a theory that FN fibril assembly precedes that of laminin.

We also performed immunoblotting assays on lysates of AEC maintained for 2 and 5 days in culture on substrates of varying stiffness using antibodies against the $\alpha 3$ laminin subunit and FN to gauge expression of these ECM components. There was barely a detectable $\alpha 3$ laminin subunit signal in immunoblots of cells maintained on substrates of lower stiffness. However, there was a significant increase in expression of this laminin subunit as a function of increasing stiffness at day 2 (Figure 5A), and this trend was calculated to be statistically significant (Figure 5B). However, the expression levels of FN were not found to be influenced by stiffness. When we analyzed the lysates from AEC harvested at day 5 , the expression levels of both the $\alpha 3$ laminin subunit and FN were found to be similar in extracts of all of our cells, regardless of the substrate stiffness on which they were maintained (Figure 5C, D).

\section{Discussion}

Traditionally, cells in culture are maintained on stiff plastic or glass substrates. Accumulating data indicate that such noncompliant surfaces have a profound impact not only on cell shape and cytoskeletal organization, but also on cell adhesion, migration, differentiation, and proliferation. ${ }^{19-22}$ The development of procedures to fabricate biologically compatible substrates of defined stiffness has allowed numerous groups to study both normal and diseased cells in vitro on surfaces or within $3 \mathrm{D}$ matrices that exhibit the physical properties of their tissue of origin. ${ }^{19,51,52}$ In this study, our goal was to assay the effects of substrate stiffness reflecting the normal and fibrotic lung on AEC adhesion and differentiation. We also wished to dissect out the role of stiffness versus matrix accumulation in determining the phenotype of AEC in a fibrotic milieu.

Substrate stiffness affects cell spreading and cytoskeleton organization of AEC. On soft substrates, AEC appear more rounded and less well spread than those cells on stiffer surfaces. Indeed, they seemed to retain many of the features of type II cells in intact lung tissue. However, despite this gross morphological appearance, in vitro AEC undergo differentiation from a type II to a type I cell type regardless of substrate stiffness. We saw no evidence that on a soft substrate, a type II phenotype was retained. Moreover, on stiff substrates, we found no evidence of enhanced EMT.
A

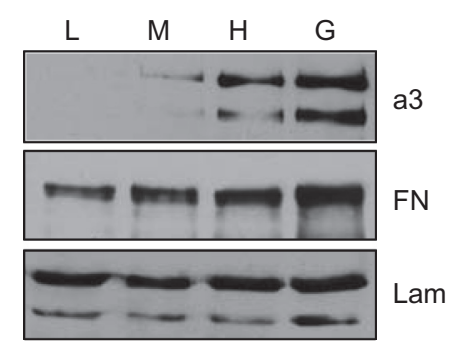

C

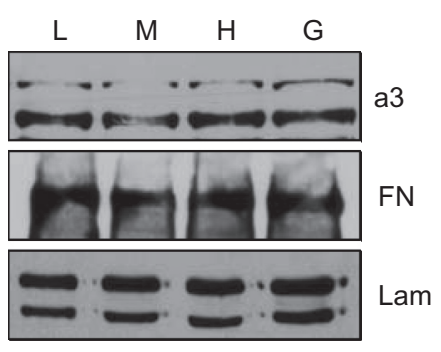

B

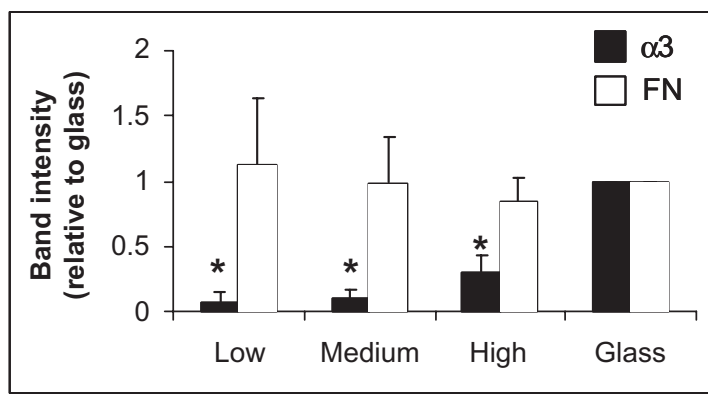

D

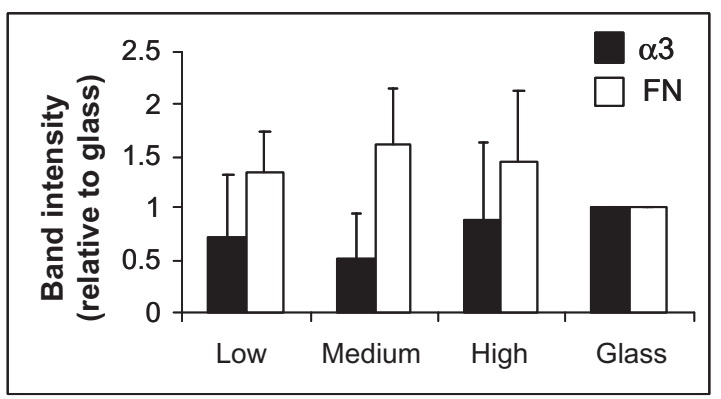

Figure 5 Representative Western blots showing the expression of the $\alpha 3$ laminin subunit, fibronectin, and lamin A/C (as a loading control) from lysates of AEC seeded onto gels and glass substrates $\mathbf{A}) 2$ days and C) 5 days postisolation. The quantification of at least three blots of each type is shown in B) and $\mathbf{D}$ ).

Note: $* P<0.05$ significance compared to the respective glass substrate.

Abbreviations: L, low stiffness gel; M, medium stiffness gel; H, high stiffness gel; G, glass substrate; $\alpha 3$, alpha3 laminin subunit; FN, fibronectin; Lam, lamin A/C. 
Together, these results suggest that substrate stiffness has no influence on AEC differentiation. However, we wish to emphasize that it is possible our soft substrates are not as compliant as the normal lung in vivo. ${ }^{31}$ We did attempt to seed AEC on gels of lower stiffness than the softest substrate tested here, but AEC failed to adhere on these very soft surfaces $(\sim 1 \mathrm{kPa})$.

An important finding of this study is that stiffness has a profound effect on laminin-311 matrix expression and assembly. Our previous work has suggested that $\alpha 3$ subunit-containing laminin fibrils in the matrix of AEC are important conduits of signals triggered during mechanical stimulation. ${ }^{5,9}$ The activation of these mechanosignaling pathways may either be protective or harmful during the extension of a damaged or diseased lung during ventilation. Here, we demonstrate that stiffness which mimics that within the fibrotic lung promotes expression of the $\alpha 3$ laminin subunit and its incorporation into a fibrillar assembly. In a recent unpublished study, we have demonstrated that a lung-specific knockout of the $\alpha 3$ laminin subunit is protective against ventilator-induced injury. Thus, we propose that the upregulation in expression of the $\alpha 3$ laminin subunit in a stiff, fibrotic environment in vivo may exacerbate disease progression.

Our data indicate that expression of $\mathrm{FN}$ is unaffected by substrate stiffness. However, substrate stiffness is an important regulator of $\mathrm{FN}$ organization in the matrix deposited by AEC. Specifically, on a stiff substrate, AEC assemble extensive arrays of FN fibrils, whereas in cultures of AEC maintained on substrates of low stiffness, we observe primarily a diffuse FN antibody staining of the extracellular matrix. The relative absence of $\mathrm{FN}$ fiber formation by AEC maintained on a compliant substrate compared with the obvious FN fibrils assembled by AEC maintained for 5 days on stiff substrates is consistent with the notion that the process of FN fibrillogenesis is determined by cellderived traction forces involving the actin cytoskeleton. These forces are promoted by a stiff substrate. ${ }^{53}$ Our data also suggest that assembly of laminin fibers containing the $\alpha 3$ subunit not only follows assembly of fibrillar FN matrix temporally, but also exhibits a precise codistribution. Indeed, we propose that laminin fibril assembly is dependent upon the assembly of a fibrillar FN matrix. Others have also predicted a link between FN and laminin assembly. ${ }^{54,55}$ Moreover, our hypothesis is supported by previous data indicating that laminin-311 fibril assembly is inhibited by a $\beta 1$ integrin blocking antibody in vitro. ${ }^{8}$ Such inhibition is also known to regulate $\mathrm{FN}$ fibrillogenesis. ${ }^{56}$

\section{Conclusions}

In conclusion, we report that although substrate stiffness influences cell adhesion and spreading, including the organization of focal adhesion structures and the actin cytoskeleton, substrate stiffness does not determine the differentiation of type II into type I alveolar cells, nor does it directly affect EMT. However, substrate stiffness significantly influences ECM organization, particularly expression of the $\alpha 3$ laminin subunit, a major component of the alveolar basement membrane within the lung. The assembly of the $\alpha 3$ laminin subunit-rich fibrillar matrix was also found to be stiffness dependent. The upregulation in expression of specific laminin subunits in AEC and the changes in matrix assembly induced by stiff substrates likely play a critical role in the progression of fibrotic diseases of the lung.

\section{Acknowledgments}

This work was supported in part by the NIH/NHBLI training grant T32HL076139 (JLE) and the NIH grant RO1HL092963 (JCRJ/GRSB). In addition, HDE acknowledges the support provided by the Office for Sponsored Research (OSR) under ONR Award N0014-08-1-0792 and by the Nanoscale Science and Engineering Initiative of the National Science Foundation (NSF) under NSF Award EEC-0647560. Microscopy was performed at the Northwestern University Cell Imaging Facility generously supported by NCI-CCSG-P30-CA060553 awarded to the Robert H Lurie Comprehensive Cancer Center. AEC isolations were performed by the Pulmonary Division Core B Facility in compliance with proper guidelines outlined by the Institutional Animal Care and Use Committee.

\section{Disclosure}

The authors report no conflicts of interest in this work.

\section{References}

1. Gonzalez R, Yang YH, Griffin C, Allen L, Tigue Z, Dobbs L. Freshly isolated rat alveolar type I cells, type II cells, and cultured type II cells have distinct molecular phenotypes. Am J Physiol Lung Cell Mol Physiol. 2005;288(1):L179-L189.

2. Danto SI, Shannon JM, Borok Z, Zabski SM, Crandall ED. Reversible transdifferentiation of alveolar epithelial cells. Am J Respir Cell Mol Biol. 1995;12(5):497-502.

3. Brody JS, Williams MC. Pulmonary alveolar epithelial cell differentiation. Annu Rev Physiol. 1992;54:351-371.

4. Shannon JM, Mason RJ, Jennings SD. Functional differentiation of alveolar type II epithelial cells in vitro: effects of cell shape, cell-matrix interactions and cell-cell interactions. Biochim Biophys Acta. 1987;931(2):143-156.

5. Jones JC, Lane K, Hopkinson SB, et al. Laminin-6 assembles into multimolecular fibrillar complexes with perlecan and participates in mechanical-signal transduction via a dystroglycan-dependent, integrinindependent mechanism. $J$ Cell Sci. 2005;118(Pt 12):2557-2566. 
6. Hamill KJ, Kligys K, Hopkinson SB, Jones JC. Laminin deposition in the extracellular matrix: a complex picture emerges. J Cell Sci. 2009; 122(Pt 24):4409-4417.

7. Aumailley M, Bruckner-Tuderman L, Carter WG, et al. A simplified laminin nomenclature. Matrix Biol. 2005;24(5):326-332.

8. DeBiase PJ, Lane K, Budinger S, Ridge K, Wilson M, Jones JC. Laminin-311 (Laminin-6) fiber assembly by type I-like alveolar cells. J Histochem Cytochem. 2006;54(6):665-672.

9. Budinger GR, Urich D, DeBiase PJ, et al. Stretch-induced activation of AMP kinase in the lung requires dystroglycan. Am J Respir Cell Mol Biol. 2008;39(6):666-672.

10. Boitano S. From the extracellular matrix to cell and tissue function in the alveolar epithelium. Am J Physiol Lung Cell Mol Physiol. 2001; 280(2):L189-L190.

11. Adamson IY, King GM, Young L. Influence of extracellular matrix and collagen components on alveolar type 2 cell morphology and function. In Vitro Cell Dev Biol. 1989;25(6):494-502.

12. Sisson TH, Mendez M, Choi K, et al. Targeted injury of type II alveolar epithelial cells induces pulmonary fibrosis. Am J Respir Crit Care Med. 2010;181(3):254-263.

13. Selman M, Pardo A. Role of epithelial cells in idiopathic pulmonary fibrosis: from innocent targets to serial killers. Proc Am Thorac Soc. 2006;3(4):364-372.

14. Cook DN, Brass DM, Schwartz DA. A matrix for new ideas in pulmonary fibrosis. Am J Respir Cell Mol Biol. 2002;27(2) $122-124$

15. Crouch E. Pathobiology of pulmonary fibrosis. Am J Physiol. 1990; 259(4 Pt 1):L159-L184

16. Kim KK, Kugler MC, Wolters PJ, et al. Alveolar epithelial cell mesenchymal transition develops in vivo during pulmonary fibrosis and is regulated by the extracellular matrix. Proc Natl Acad Sci USA. 2006;103(35):13180-13185.

17. Willis BC, Liebler JM, Luby-Phelps K, et al. Induction of epithelialmesenchymal transition in alveolar epithelial cells by transforming growth factor-beta1: potential role in idiopathic pulmonary fibrosis. Am J Pathol. 2005;166(5):1321-1332.

18. Kasai H, Allen JT, Mason RM, Kamimura T, Zhang Z. TGF-beta1 induces human alveolar epithelial to mesenchymal cell transition (EMT). Respir Res. 2005;6:56.

19. Discher DE, Janmey P, Wang YL. Tissue cells feel and respond to the stiffness of their substrate. Science. 2005;310(5751):1139-1143.

20. Yeung T, Georges PC, Flanagan LA, et al. Effects of substrate stiffness on cell morphology, cytoskeletal structure, and adhesion. Cell Motil Cytoskeleton. 2005;60(1):24-34.

21. Janmey PA, Winer JP, Murray ME, Wen Q. The hard life of soft cells. Cell Motil Cytoskeleton. 2009;66(8):597-605.

22. Chen CS, Tan J, Tien J. Mechanotransduction at cell-matrix and cell-cell contacts. Annu Rev Biomed Eng. 2004;6:275-302.

23. Bhana B, Iyer RK, Chen WL, et al. Influence of substrate stiffness on the phenotype of heart cells. Biotechnol Bioeng. 2010;105(6): 1148-1160.

24. Leipzig ND, Shoichet MS. The effect of substrate stiffness on adult neural stem cell behavior. Biomaterials. 2009;30(36):6867-6878.

25. Even-Ram S, Artym V, Yamada KM. Matrix control of stem cell fate. Cell. 2006;126(4):645-647.

26. Lee JN, Jiang X, Ryan D, Whitesides GM. Compatibility of mammalian cells on surfaces of poly(dimethylsiloxane). Langmuir. 2004;20(26): 11684-11691.

27. Venkatesh SK, Yin M, Glockner JF, et al. MR elastography of liver tumors: preliminary results. AJR Am J Roentgenol. 2008;190(6): 1534-1540.

28. Gang Z, Qi Q, Jing C, Wang C. Measuring microenvironment mechanical stress of rat liver during diethylnitrosamine induced hepatocarcinogenesis by atomic force microscope. Microsc Res Tech. 2009 72(9):672-678.

29. Faffe DS, Zin WA. Lung parenchymal mechanics in health and disease. Physiol Rev. 2009;89(3):759-775.
30. Maksym GN, Bates JH. A distributed nonlinear model of lung tissue elasticity. J Appl Physiol. 1997;82(1):32-41.

31. Liu F, Mih JD, Shea BS, et al. Feedback amplification of fibrosis through matrix stiffening and COX-2 suppression. J Cell Biol. 2010; 190(4):693-706.

32. Wang YL, Pelham RJ Jr. Preparation of a flexible, porous polyacrylamide substrate for mechanical studies of cultured cells. Methods Enzymol. 1998;298:489-496.

33. Butt HJ, Cappella B, Kappl M. Force measurements with the atomic force microscope: technique, interpretation and applications. Surf Sci Rep. 2005;59(1-6):1-152.

34. Sader JE, Larson I, Mulvaney P, White LR. Method for the calibration of atomic force microscope cantilevers. Rev Sci Instrum. 1995;66(7): 3789-3798.

35. Oliver WC, Pharr GM. An improved technique for determining hardness and elastic modulus using load and displacement sensing indentation experiments. J Mater Res. 1992;7(6):1564-1583.

36. Fischer-Cripps AC. Nanoindentation. 2nd ed. Berlin, Germany: Springer-Verlag; 2004.

37. Goldfinger LE, Jiang L, Hopkinson SB, Stack MS, Jones JC. Spatial regulation and activity modulation of plasmin by high affinity binding to the $\mathrm{G}$ domain of the alpha 3 subunit of laminin-5. J Biol Chem. 2000;275(45):34887-34893.

38. Baker SE, Hopkinson SB, Fitchmun M, et al. Laminin-5 and hemidesmosomes: role of the alpha 3 chain subunit in hemidesmosome stability and assembly. J Cell Sci. 1996;109(Pt 10):2509-2520.

39. Damljanović V, Lagerholm BC, Jacobson K. Bulk and micropatterned conjugation of extracellular matrix proteins to characterized polyacrylamide substrates for cell mechanotransduction assays. Biotechniques 2005;39(6):847-851.

40. Engler AJ, Richert L, Wong JY, Picart C, Discher DE. Surface probe measurements of the elasticity of sectioned tissue, thin gels and polyelectrolyte multilayer films: correlations between substrate stiffness and cell adhesion. Surf Sci. 2004;570(1-2):142-154.

41. Solon J, Levental I, Sengupta K, Georges PC, Janmey PA. Fibroblast adaptation and stiffness matching to soft elastic substrates. Biophys $J$. 2007;93(12):4453-4461

42. Constantinides G, Kalcioglu ZI, McFarland M, Smith JF, van Vliet KJ. Probing mechanical properties of fully hydrated gels and biological tissues. J Biomech. 2008;41(15):3285-3289.

43. Van Landingham MR, Villarrubia JS, Guthrie WF, Meyers GF. Nanoindentation of polymers: an overview. Macromol Symp. 2001; 167(1):15-44

44. Tranchida D, Piccarolo S. Combining atomic force microscopy and depth-sensing instruments for the nanometer-scale mechanical characterization of soft matter. In: Bhushan B, editor. Scanning Probe Microscopy in Nanoscience and Nanotechnology. New York, NY: Springer; 2010:199-223.

45. Engler AJ, Sen S, Sweeney HL, Discher DE. Matrix elasticity directs stem cell lineage specification. Cell. 2006;126(4):677-689.

46. Hynes RO. Integrins: bidirectional, allosteric signaling machines. Cell. 2002;110(6):673-687.

47. Berrier AL, Yamada KM. Cell-matrix adhesion. J Cell Physiol. 2007; 213(3):565-573.

48. Geiger B, Spatz JP, Bershadsky AD. Environmental sensing through focal adhesions. Nat Rev Mol Cell Biol. 2009;10(1):21-33.

49. Lee JM, Dedhar S, Kalluri R, Thompson EW. The epithelialmesenchymal transition: new insights in signaling, development, and disease. J Cell Biol. 2006;172(7):973-981.

50. Kalluri R. EMT: when epithelial cells decide to become mesenchymallike cells. J Clin Invest. 2009;119(6):1417-1419.

51. Buxboim A, Ivanovska IL, Discher DE. Matrix elasticity, cytoskeletal forces and physics of the nucleus: how deeply do cells 'feel' outside and in? J Cell Sci. 2010;123(Pt 3):297-308.

52. Moore SW, Roca-Cusachs P, Sheetz MP. Stretchy proteins on stretchy substrates: the important elements of integrin-mediated rigidity sensing. Dev Cell. 2010;19(2):194-206. 
53. Lemmon CA, Chen CS, Romer LH. Cell traction forces direct fibronectin matrix assembly. Biophys J. 2009;96(2):729-738.

54. Schwarzbauer JE, Sechler JL. Fibronectin fibrillogenesis: a paradigm for extracellular matrix assembly. Curr Opin Cell Biol. 1999;11(5): $622-627$.

55. Austria MR, Couchman JR. Enhanced assembly of basement membrane matrix by endodermal cells in response to fibronectin substrata. $J$ Cell Sci. 1991;99(Pt 2):443-451.
56. Sechler JL, Cumiskey AM, Gazzola DM, Schwarzbauer JE. A novel RGD-independent fibronectin assembly pathway initiated by alpha4beta 1 integrin binding to the alternatively spliced V region. J Cell Sci. 2000;113(Pt 8):1491-1498.

\section{Publish your work in this journal}

Research and Reports in Biology is an international, peer-reviewed, open access journal publishing original research, reports, editorials, reviews and commentaries on all areas of biology including animal biology, biochemical biology, cell biology, ecological studies, evolutionary biology, molecular biology, plant science and botany. The manuscript management system is completely online and includes a very quick and fair peer-review system. Visit http://www.dovepress. com/testimonials.php to read real quotes from published authors.

Submit your manuscript here: http://www.dovepress.com/research-and-reports-in-biology-journal 